\title{
Charles de Gaulle visto dall'Italia (1958-2012)
}

Charles de Gaulle vu de l'Italie (1958-2012)

Charles de Gaulle Seen from Italy (1958-2012)

Roberto Colozza

\section{(2) OpenEdition}

\section{Journals}

Edizione digitale

URL: http://journals.openedition.org/cei/2892

DOI: 10.4000/cei.2892

ISSN: 2260-779X

\section{Editore}

UGA Éditions/Université Grenoble Alpes

Edizione cartacea

Data di pubblicazione: 20 avril 2016

Paginazione: $97-110$

ISBN: 978-2-84310-323-0

ISSN: $1770-9571$

\section{Notizia bibliografica digitale}

Roberto Colozza, «Charles de Gaulle visto dall'Italia (1958-2012)», Cahiers d'études italiennes [Online] 22 | 2016, online dal 01 janvier 2017, consultato il 26 mars 2021. URL: http://journals.openedition.org/ cei/2892 ; DOI: https://doi.org/10.4000/cei.2892 
CHARLES DE GAULLE VISTO DALL'ITALIA (1958-2012)

\author{
Roberto Colozza \\ Gerda Henkel Stiftung
}

La percezione del 'fenomeno' Charles de Gaulle si compone di vari elementi, che sono espressione di un profilo biografico in cui la dimensione personale si mescola pressoché inscindibilmente con quella politica. Analizzare la ricezione di de Gaulle in Italia significa tener presente questo palinsesto di realtà. Assecondando quello stesso processo di personalizzazione della politica che de Gaulle perseguiva programmaticamente e, verrebbe da dire, istintivamente, il giudizio su di lui tende a unificare in un'unica percezione l'uomo e lo statista. Le opinioni della classe politica italiana su de Gaulle sono in parte note, soprattutto con riferimento al periodo immediatamente successivo al ritorno al potere del Generale nel 1958. È per questo che il presente contributo allarga l'arco cronologico dell'analisi alla ricerca di punti di vista meno esplorati, ma non meno indicativi di un dibattito che ha attraversato la storia recente italiana senza soluzione di continuità. Sebbene il tema centrale del mio studio riguardi la ricezione di de Gaulle presso gli ambienti politici italiani, ho ritenuto opportuno concedermi incursioni anche in quelli accademici e nella letteratura scientifica, giacché in qualche caso ricerca e militanza coesistono e si alimentano vicendevolmente.

L'ascesa di de Gaulle al governo nel 1958 fu considerata dalla maggioranza del mondo politico italiano come un evento potenzialmente pericoloso per la stabilità della democrazia francese. Militare di carriera, oppositore noto della Quarta Repubblica, la cui Costituzione aveva non pochi punti in comune con quella della giovane Repubblica italiana ${ }^{\mathrm{I}}$, de Gaulle appariva a molti come l'emissario delle frange nazionaliste desiderose di

I. J. Rivero, Constitution italienne et Constitution française, in E. Crosa (a cura di), La Constitution italienne de 1948, "Cahiers de la Fondation nationale des sciences politiques», nº I8, Paris, Colin, I950, pp. IX-XVII. 
conservare l'Algeria francese, o comunque come un uomo fondamentalmente affine a quelle lobby e disposto ad accettarne le istanze. Questo sguardo sospettoso verso de Gaulle era naturalmente molto presente a sinistra.

Il segretario generale del Pci Palmiro Togliatti pensava che de Gaulle ponesse le basi di una deriva autoritaria, eventualmente di un vero e proprio fascismo alla francese ${ }^{2}$. Nonostante estemporanei apprezzamenti per le posizioni geopolitiche del Generale - ostile verso la Ced e la Nato, freddo nei riguardi degli Usa e aperto a un'idea d'Europa estesa oltre la cortina di ferro della Guerra fredda - la percezione del gollismo presso il Pci resterà sostanzialmente quella di un partito ostile per ideologia e habitus al nuovo leader francese ${ }^{3}$. Giudizi negativi circolavano anche nel Psi, soprattutto nell'ala sinistra del partito. Si pensi a Lelio Basso, ex segretario generale tra il gennaio 1947 e l'aprile 1948 e pensatore molto influente nonostante l'eterodossia rispetto ai canoni del frontismo filosovietico in voga. Basso non identificava l'avvento di de Gaulle con l'inizio di un vero e proprio fascismo alla francese; tuttavia non aveva dubbi nel considerare il Generale l'avanguardia della 'reazione' transalpina e, se non un dittatore, comunque la testa di ponte per una deriva dittatoriale. Allargando la prospettiva d'analisi alla longue durée, Basso riconduceva il ritorno al potere di de Gaulle entro i binari di una prassi francese: quella di affidare a un salvatore, spesso un militare, le sorti del Paese nei momenti di maggiore crisi. Sedicente "patria della democrazia», la Francia, arguiva Basso, era in realtà una «democrazia governata» da una pubblica amministrazione onnipotente, non una «democrazia governante» dove il potere decisionale fosse effettivamente nelle mani del popolo sovrano secondo i crismi definitori di Georges Burdeau ${ }^{4}$.

A proposito di sinistra italiana e de Gaulle, impossibile non richiamare la citatissima conferenza di Gilles Martinet alla Casa della Cultura di Milano. Diretta da Rossana Rossanda, funzionaria comunista destinata a rapida ascesa nelle gerarchie del partito prima della nota deriva eterodossa sfociata nella fondazione de «il Manifesto», la Casa della Cultura era

2. P. Togliatti, Le 'vie nazionali' del fascismo, in Togliatti e il centrosinistra, 1958-1964, vol. I, Firenze, Istituto Gramsci di Firenze, 1975, pp. 28 sgg.

3. G. Pécout, "Les pressions de la France gaulliste». Les communistes italiens et la politique européenne du général de Gaulle : le regard d'Emilio Sereni et de Gerardo Chiaromonte, in De Gaulle et l'Italie, actes du colloque (Rome, Ir -3 mars 1990), Roma, École française de Rome, I997, pp. I69-20I.

4. Basso traeva questi concetti da G. Burdeau, La Démocratie gouvernante. Ses structures gouvernementales, Paris, LGDJ, 1957. Sulla posizione di Basso in merito alla crisi francese, cfr. L. Basso, La crisi della democrazia francese e le sue cause (I-3), "Problemi del socialismo", $\mathrm{n}^{\circ}$ 6, giugno I958, pp. 407-426; $\mathrm{n}^{\circ}$ 7, luglio I958, pp. 487$505 ; \mathrm{n}^{\circ} 8$, agosto 1958 , pp. 567-588. 
all'epoca lo specchio della variegata gamma ideologica della sinistra antigovernativa italiana. Nonostante il titolo della conferenza — «La Francia diventerà fascista?» - lasciasse presagire altro, Martinet impostò un'analisi circostanziata e prudente in cui prevaleva il desiderio di comprendere più che di giudicare i fatti francesi. Ma l'uditorio italiano, convinto delle mire irrimediabilmente autocratiche del generale, sembrò disorientato, e l'incontro finì per naufragare nell'incomunicabilità'.

Questa sfiducia veniva certo dal retroterra culturale della sinistra italiana, egualitario e parlamentarista, ma derivava anche dal suo rapporto speciale con la Costituzione repubblicana. Il Pci, in particolare, si sentiva araldo del patto fondatore della Repubblica, sul quale aveva investito gran parte della propria credibilità, effettiva o strumentale che fosse, rispetto agli standard della democrazia liberale. La difesa della Costituzione fondata sulla Resistenza antifascista fu il pilastro del discorso comunista sulla nazione e sulla democrazia fino alla fine della Guerra fredda. Se è forse esagerato parlare di «mito della Costituzione» ${ }^{6}$, è però legittimo riconoscere che nella cultura comunista - e con modi comunicativi, contenuti e finalità diversi anche in quelle azionista e socialista — la Costituzione repubblicana ha preso talora le sembianze di una bandiera. Il che ha impedito a volte una discussione costruttiva intorno ai modi per aggiornare la Carta e adattarla all'evolversi della Repubblica. D'altra parte proprio l'opposizione 'socialcomunista' e l'azionismo culturale furono protagonisti negli anni Cinquanta di una campagna rivendicativa volta ad attuare principi e istituti previsti dalla Carta. Una campagna, quella per l'attuazione della Costituzione, che fu minoritaria, coraggiosa e utile a sbloccare l'impasse normativa dei governi centristi, a loro volta preoccupati di non 'scongelare' anzitempo la Costituzione per non concedere troppa libertà d'azione alle sinistre antisistema ${ }^{7}$.

D'altronde l'esperienza della dittatura fascista aveva reso la classe dirigente italiana ostile verso ogni forma di personalizzazione della politica, cosa che condizionava la valutazione anche presso osservatori non prevenuti ideologicamente verso il Generale. Si può citare il caso di Costantino Mortati, costituzionalista e membro dell'Assemblea costituente nei ranghi

5. G. Martinet, Cassandre et les tueurs. Cinquante ans d'une histoire française, Paris, Grasset, 1986, pp. I28I29. L'episodio è citato anche in G. Martinet e S. Romano, Une amitié difficile. Entretiens sur deux siècles de relations franco-italiennes, Paris, Association Dante Alighieri-Comité de Paris, 1999, p. 78.

6. E. Galli della Loggia, Il mito della Costituzione, in G. Belardelli et al., Miti e storia dell'Italia unita, Bologna, Il Mulino, I999, pp. I87-20I.

7. U. De Siervo, La mancata riforma delle istituzioni politiche, in U. De Siervo, S. Guerrieri e A. Varsori (a cura di), La prima legislatura. Continuità e discontinuità nell'azione delle istituzioni, Roma, Carocci, 2004. 
della Dc; di Giuseppe Saragat, leader del Psdi e futuro presidente della Repubblica; di Luigi Gui, anch'egli ex costituente, dirigente della Dc e prossimo ministro nei governi di centro-sinistra; e di Luigi Sturzo, sacerdote siciliano e fondatore del Partito popolare italiano, antesignano della $\mathrm{Dc}^{8}$. Lo stesso ambasciatore francese a Roma, Gaston Palewski, raccolse scarsi risultati quando, durante un incontro a palazzo Farnese, cercò di veicolare un'immagine meno apocalittica degli eventi francesi del maggio I958 rispetto a quella che si andava affermando agli occhi degli intellettuali italiani ${ }^{9}$. Né la vittoria nel referendum del settembre 1958 da parte di de Gaulle e della sua idea di costituzione, già orientata verso un'originale forma di semipresidenzialismo, poteva in alcun modo far prevalere nella percezione degli osservatori italiani i meriti del Generale piuttosto che i rischi legati alla sua concezione delle istituzioni. Le critiche suscitate in Italia dall'avvento al potere di de Gaulle seguirono un andamento piuttosto costante, rinfocolato dalla riforma costituzionale del 1962. In Francia fu questo l'evento che catalizzò i sentimenti di rigetto verso il Generale. Dopo aver ottenuto la pace con gli accordi di Evian, de Gaulle sembrava infatti abusare del suo potere politico e mediatico. Anziché ritirarsi in buon ordine dopo aver compiuto la propria missione e risolto l'emergenza algerina, de Gaulle pareva rilanciare la posta in gioco cercando il consenso per accentrare ulteriormente il potere nelle proprie mani.

In questo panorama di pareri piuttosto omogeneo, una posizione sfumata era quella di Amintore Fanfani. Lungi dall'immaginare una soluzione di tipo gollista all'instabilità politica e al proliferare dei partiti in Italia, Fanfani stimava de Gaulle e lo considerava una personalità adatta al contesto transalpino. L'ammirazione dello statista aretino per il Generale era almeno in parte ricambiata, se è vero che proprio Fanfani fu il primo capo di governo straniero ricevuto da de Gaulle dopo il suo ritorno al potere e che l'accoglienza riservatagli travalicò la cordialità protocollare prevista negli incontri ufficiali. Non mancarono nel dibattito italiano voci che sottolineavano le somiglianze tra i due leader: in senso negativo - è il caso del Pci, che mirava a screditare il segretario democristiano descrivendolo appunto come un possibile mattatore autoritario; in senso positivo - come negli ambienti diplomatici francesi a Roma e in settori influenti dell'amministrazione statunitense. Giorgio La Pira, sindaco di Firenze e

8. Si veda in proposito P. Scoppola, La repubblica dei partiti. Evoluzione e crisi di un sistema politico, I9451996, Bologna, Il Mulino, I99I, pp. 34I sgg. Quanto alla posizione di Sturzo, cfr. in particolare L. Sturzo, L'Italia e de Gaulle, in Id., Tre male bestie, Napoli, Ed. politica popolare, 1959.

9. G. Palewski, Mémoires d'action, I924-1974, Paris, Plon, I988, pp. 273-274. 
noto propugnatore di un'idea di democrazia umanitaria e spirituale, appoggiava entusiasticamente il Generale perché vedeva in lui l'unica possibile figura capace di restaurare il prestigio della Francia cattolica e di porla in prima linea nella costruzione di un'area mediterranea basata sul dialogo tra culture piuttosto che su conflitti vetero-colonialisti del genere di quello che la stessa Francia combatteva ormai da quattro anni in Algeria. Queste forme di apprezzamento verso de Gaulle da parte di Fanfani e La Pira non si accompagnarono a tentativi duraturi di collaborazione politico-diplomatica visto che il prevalente atteggiamento filoarabo della Dc e il sospetto francese che Enrico Mattei, tramite l'Eni, finanziasse il Fronte di liberazione nazionale algerino in lotta con Parigi costituivano ostacoli insormontabili a eventuali progetti d'intesa tra i due governi ${ }^{\text {Io. }}$

Fuori dalla Dc, gli estimatori di de Gaulle militavano essenzialmente nella destra neofascista - è il caso di Giorgio Pisanò, ex repubblichino, tra i fondatori del Msi e dichiarato ammiratore della riforma costituzionale francese del 1962 - o tra i cattolici conservatori come Gianni Baget

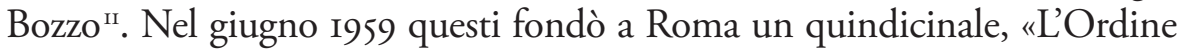
civile», che per un anno e mezzo fu tra le più lucide voci a favore di una svolta di tipo presidenziale nell'Italia dell''apertura a sinistra' ${ }^{\text {'2 }}$. Un'eccezione rimarchevole nel novero degli ammiratori di de Gaulle è incarnata da Randolfo Pacciardi, singolare figura di repubblicano, mazziniano, antifascista e anticomunista, ex combattente volontario a difesa della Repubblica spagnola, quando assunse il comando del Battaglione (poi Brigata) Garibaldi. Pacciardi fondò nel 1964 il movimento d'Unione democratica per la Nuova Repubblica (Udnr), avente come fine primario una riforma costituzionale che introducesse il presidenzialismo in Italia e che guardava alla Quinta Repubblica come a un esempio costruttivo.

La stima personale di Pacciardi verso de Gaulle non era in discussione e prese le mosse dall'appello del I8 giugno 1940, sfociando poi in una richiesta di collaborazione alla France Libre risoltasi in un nulla di fatto. L'occupazione francese della Val d'Aosta sotto il governo provvisorio del Generale fu vissuta da Pacciardi come una ferita all'orgoglio nazionale dell'Italia antifascista e alla sorellanza latina, e incrinò in lui il mito de Gaulle. Quando nel 1947 il Generale fondò il Rassemblement du peuple

Io. A. Argenio, Da maggio a maggio. La Dc e l'avvento al potere di Charles de Gaulle nel 1958, "Italia contemporanea», $\mathrm{n}^{\circ} 27 \mathrm{O}$, marzo 2013 , pp. I2-32.

II. R. Chiarini, La fortuna del gollismo in Italia. Le suggestioni di una 'Seconda Repubblica', "Storia contemporanea", $\mathrm{n}^{\circ}$ 2, 1994, pp. 173-220.

I2. Lo stesso Baget Bozzo tratteggia una breve storia della sua rivista in Il partito cristiano e l'apertura a sinistra. La Dc di Fanfani e di Moro, I954-1962, Firenze, Vallecchi, 1977, pp. 193-198. 
français (Rpf) Pacciardi rimase tiepido e stigmatizzò le mire a suo dire reazionarie e bonapartiste del nuovo partito. I fatti del 1958 riaccesero la fede di Pacciardi in de Gaulle; una visione che rimase prevalente nella percezione dell'uomo fino alle dimissioni del Generale nel 1969. Ciononostante, la pubblicistica prodotta dall'Udnr lascia trasparire una chiara preferenza per il presidenzialismo statunitense. La personalizzazione del potere instaurata in Francia con la Quinta Repubblica era certo uno strumento di governo apprezzabile ma non era sostenibile sul lungo periodo, perché enfatizzava le capacità dirigenziali di un uomo e metteva in secondo piano un aspetto ben più importante per la buona salute delle istituzioni: ovvero il loro legame con la società civile, le forze produttive, i sindacati; una dimensione, questa, che il sistema statunitense sembrava realizzare in modo più efficace $^{\text {13 }}$. La predilezione di Pacciardi verso l'assetto istituzionale statunitense è confermata, perfino enfatizzata, dalle dichiarazioni dell'uomo politico italiano al cospetto della rappresentanza diplomatica Usa nella fase di genesi dell'Udnr ${ }^{14}$.

Le voci italiane non ostili a de Gaulle, pur eterogenee, avevano in comune l'intento di porre fine a quello che lo stesso generale chiamava il 'régime des partis', ovvero la democrazia parlamentare come si era configurata durante la Quarta Repubblica e come si affermava ormai a sud delle Alpi. A questo 'regime' fu dato in Italia il nome di 'partitocrazia', un termine oggi entrato nel linguaggio comune e coniato dal liberale monarchico Roberto Lucifero in un volume del $1944^{\text {I5 }}$. Impiegato anche da Benedetto Croce e Arturo Labriola, il neologismo conobbe la sua consacrazione accademica grazie a Giuseppe Maranini, che lo eresse ad autentica categoria interpretativa della storia repubblicana ${ }^{16}$. Non a caso l'etichetta di 'partitocrazia' conobbe un significativo sviluppo durante gli anni Sessanta, in opposizione ai governi di centro-sinistra basati sull'alleanza tra Dc e Psi, unica verosimile via d'uscita dall'impasse in cui il fallimento della 'legge truffa' e la crisi del centrismo avevano costretto il paese. Gli

13. M. Mita, La repubblica di domani, Edizioni Folla, novembre 1964; Id., Il regime presidenziale, Edizioni dell'Udnr, 1967; Id., Costruire la Repubblica, Roma, Nuova Repubblica, 1968. Su Pacciardi vedi P. Palma, Randolfo Pacciardi. Profilo politico dell'ultimo mazziniano, Soveria Mannelli, Rubbettino, 20I2, pp. 67-73.

I4. Cfr. l'aerogramma confidenziale (Io febbraio 1964) dell'ambasciata Usa di Roma al Dipartimento di Stato sulla conversazione avuta da Pacciardi con funzionari diplomatici statunitensi il 4 febbraio 1964, in M. Franzinelli e A. Giacone (a cura di), Il riformismo alla prova. Il primo governo Moro nei documenti e nelle parole dei protagonisti (ottobre I963-agosto I964), "Annali della Fondazione Giangiacomo Feltrinelli», $\mathrm{n}^{\circ} 46$, 20I0, Milano, Feltrinelli, 20I2, p. I90-I94. Ringrazio Alessandro Giacone per avermi segnalato il documento e avermene fornito copia.

I5. R. Lucifero, Introduzione alla libertà (La legge elettorale), Roma, Ed. del Secolo, I944.

16. E. Capozzi, Partitocrazia. Il regime italiano e i suoi critici, Napoli, Guida, 2009, pp. 43 sgg. 
'antipartitocratici' leggevano in questa combinazione parlamentare la definitiva prova di un'attitudine trasformistica nella classe dirigente italiana che impediva un effettivo riformismo e soffocava le istituzioni sotto la cappa degli appetiti di partito e delle convenienze contingenti.

D'altronde il successo della 'partitocrazia' italiana è dato dal fatto che questa soddisfaceva le esigenze di filtro tra società civile e istituzioni con un'efficienza che entrerà in crisi solo sul finire degli anni Sessanta — si pensi al Sessantotto e all'inizio dei cosiddetti 'anni di piombo'. Rispetto alla realtà italiana, i partiti francesi del dopoguerra si caratterizzavano per una maggiore elasticità degli apparati organizzativi e per una minore capacità d'influenzare le dinamiche della società civile. Priva di esperienze marcanti e durature come il totalitarismo fascista, la Quarta Repubblica si era sviluppata secondo modalità assimilabili a quelle che avevano già connotato la Terza Repubblica: partiti politici relativamente leggeri, con bacini d'iscritti minori rispetto al caso italiano. Il processo di decolonizzazione, che l'Italia non dovette affrontare, diede il colpo di grazia a questo sistema tutto sommato fragile. In altre parole, la 'partitocrazia' italiana era certo più confacente al paese di quanto non fosse il 'régime de partis' stigmatizzato da de Gaulle, e questo indipendentemente dal fatto che in Francia esistesse una credibile alternativa al parlamentarismo; cosa che non si può dire valesse per l'Italia.

E tuttavia vi fu chi tentò di mettere in discussione l'egemonia del patto parlamentarista attraverso un abbozzo di semipresidenzialismo. Nel 1955 l'ascesa di Giovanni Gronchi al Quirinale aveva promosso al vertice dello Stato un esponente 'illuminato' della Dc, ben accolto anche in ambienti lontani o ostili al partito di maggioranza, in particolare a sinistra. Grazie all'approccio decisionista del nuovo capo dello Stato e a una congiuntura che virava alla distensione, furono introdotti in poco tempo vari istituti fondamentali previsti dalla Carta repubblicana, come ad esempio la Corte costituzionale. Proprio Gronchi è al centro di un episodio indicativo del crescente desiderio di riforma che serpeggiava nei palazzi romani sul finire degli anni Cinquanta. La vicenda coinvolge il giudice della Corte costituzionale Mario Bracci, già rettore dell'Università di Siena e vicino al Psi, dunque a un partito tradizionalmente marcato da un rigoroso parlamentarismo. In virtù della confidenza amicale che lo accomunava a Gronchi, Bracci scrisse al capo dello Stato una lettera privata a breve distanza dal referendum francese del 1958. Bracci non guardava a de Gaulle e alla soluzione francese come a uno sbocco auspicabile per l'Italia, ma riteneva che la situazione italiana fosse molto diversa da quella transalpina, non solo per l'assenza di crisi esiziali come quella algerina ma anche per una maggiore 
solidità della Costituzione, protetta da un sapiente sistema di checks and balances. Certo il logoramento delle istituzioni repubblicane imponeva un'assunzione di responsabilità da parte di una figura istituzionale di vertice, e questo compito non poteva essere che del capo dello Stato.

Bisogna spostare il regime, nei limiti consentiti dall'interpretazione della Costituzione, e, per quanto sia politicamente possibile, dalla tradizionale prevalenza del Parlamento, spesso velleitaria, alla prevalenza del Presidente della Repubblica, verso quel tipo originale di repubblica presidenziale che è reso possibile dalla lettera e dallo spirito della Costituzione $[\ldots]$.

Diversamente da quanto avvenuto in Francia, in Italia, secondo Bracci, il rafforzamento del presidente della Repubblica non implicava una riforma costituzionale ma solo un'accentuazione di prerogative già previste dalla Carta. Il controllo esercitato sul presidente dalla Corte costituzionale avrebbe scongiurato il rischio di derive autocratiche. Un presidente così concepito avrebbe potuto esercitare una funzione d'indirizzo carismatica, che passasse anche attraverso un'accentuata presa mediatica sulla popolazione. Anticipando quel che de Gaulle realizzerà durante la sua lunga esperienza da capo dello Stato, Bracci individuava nei messaggi presidenziali attraverso TV e radio la chiave di volta per instaurare un rapporto diretto tra presidente e cittadini, sviluppando così le peculiarità migliori del regime presidenziale ${ }^{17}$.

Questo tentativo d'interpretazione della Costituzione rimarrà circoscritto alle 'stanze dei bottoni', condiviso da alcune figure isolate ma avversato dai vertici dei partiti, che sempre più si configuravano come i principali elementi decisori del sistema e non erano certo disposti ad accogliere una logica istituzionale estranea al loro orizzonte programmatico, oltre che ai loro interessi. Fuori dai partiti, Luigi Sturzo sostenne l'incompatibilità tra il modello istituzionale suggerito da Gronchi ed il contesto italiano, inadatto a conciliare principio democratico ed istituzioni a forte tasso personalistico. In riferimento al capo dello Stato in carica, ma senza citarlo, Sturzo scriveva: «[...] si vorrebbero surrettiziamente inserire metodi, interferenze e atti non previsti dalla Costituzione, purché tollerati dai partiti favorevoli; i quali diverrebbero, per il tramite dei gruppi parlamentari, una specie di elettori pretoriani» ${ }^{18}$.

17. M. Bracci, Testimonianze sul proprio tempo. Meditazioni, lettere, scritti politici (1943-I958), a cura di E. Balocchi e G. Grottanelli de’ Santi, Firenze, La Nuova Italia, I98I, pp. 729 e 740. Per un'analisi del ruolo dei media nella costruzione del carisma gollista, R. Brizzi, L’uomo dello schermo. De Gaulle e i media, Bologna, Il Mulino, 20I0.

18. L. Sturzo, Tre male bestie, cit., p. 94. 
Sull'altro versante della barricata, una significativa sponda forniva agli aspiranti riformatori il politologo Giuseppe Maranini. Poco influente a causa della sua eccentricità ideologica ma intellettualmente autorevole per competenza e posizione gerarchica nella nomenclatura universitaria, Maranini operava in un contesto accademico che, proprio come il mondo politico, vedeva nella Francia di de Gaulle un anti-modello piuttosto che una fonte d'ispirazione ${ }^{19}$. E anche Maranini, come Bracci, era convinto che la Costituzione italiana non avesse bisogno di essere riformata ma che il capo dello Stato potesse tramite il semplice menage istituzionale acquisire una preminenza di fatto rispetto al parlamento e quindi ai partiti.

Pur riconoscendo al Generale il merito di aver fornito una risposta alla crisi algerina, neppure Maranini apprezzava il presidenzialismo à la de Gaulle, che non gli sembrava risolvere i problemi atavici della Francia post-rivoluzionaria: assemblearismo, conflitto tra i poteri dello Stato, scarsa autonomia del potere esecutivo nei confronti del legislativo. Per rispondere a queste deficienze, de Gaulle aveva annichilito il parlamento e neutralizzato il governo, investendo il capo dello Stato di un potere debordante: tutto il contrario dell'assetto equilibrato del presidenzialismo statunitense, cui Maranini guardava come a un sistema armonioso capace di frazionare e distribuire potere senza ricorrere a forme di cesarismo plebiscitario. Maranini collaborò con l'Undr e prestò la propria consulenza per la stesura dell' «Appello per la Nuova Repubblica» edito nel febbraio 1965. Le sue frequentazioni con Pacciardi smussarono la freddezza con cui Maranini aveva guardato a de Gaulle, ma ciò non lo indusse né ad aderire all'Udnr né a parteggiare per il modello repubblicano gollista, che restò ai suoi occhi un esito straordinario e precario rispetto a una situazione di crisi che nasceva da squilibri cronici nell'interazione tra i poteri statuali ${ }^{20}$.

Per Pacciardi, invece, la vicinanza con Maranini costituiva un'utilissima mallevadoria per il proprio progetto politico, che poteva così fregiarsi dell'illustre avallo di un intellettuale di rango. Si veda in proposito quanto Pacciardi disse durante un discorso al teatro Adriano di Roma nel maggio $1964^{2 \mathrm{I}}$, arringando i presenti contro la "partitocrazia italiana» giunta al suo apice, secondo lui, col primo governo di centro-sinistra:

19. Si veda in merito la breve disamina di C. Fusaro, L'Italia e il modello della Quinta Repubblica: dall'odio, all'amore... e al dubbio, consultabile al seguente indirizzo web: <www.forumcostituzionale.it/wordpress/wpcontent/uploads/pre_2006/773.pdf>.

20. E. Capozzi, Il sogno di una costituzione. Giuseppe Maranini e l'Italia del Novecento, Bologna, Il Mulino, 2004, pp. 266-276.

2I. R. Pacciardi, Una Repubblica da riformare, in Id., Randolfo Pacciardi, Roma, Camera dei deputati Archivio storico, 20II, pp. 215-216. 
Hanno protestato i costituzionalisti. Ce n'è uno a Firenze, il preside della facoltà di scienze politiche che ha concepito la cattedra universitaria come dovrebbero essere concepite tutte le cattedre universitarie italiane, come centri di irradiazione di scienza e di sapere. Parlo di Maranini che sui giornali fa una campagna sostenuta contro questo sistema della partitocrazia e lo seguono i suoi allievi diventati a loro volta professori di università.

"Ci sono i giornalisti che protestano di tanto in tanto», proseguiva ancora Pacciardi, «ci sono i senatori e i deputati che negli articoli dei giornali protestano anch'essi e poi votano...». Come si può notare, Pacciardi disegnava una sorta di mappa delle sparute forze 'antipartitocratiche', auspicando implicitamente una vera e propria controffensiva contro il sistema dei partiti, che sarebbe dovuta passare attraverso le aule universitarie, le grandi testate giornalistiche e naturalmente le istituzioni statuali. La realtà italiana, come detto, mostrava un clima ben poco adatto ad azioni del genere e soprattutto a soluzioni riformatrici calcate sull'esempio d'oltralpe.

La perplessità riguardo alle idee istituzionali di de Gaulle non era certo limitata al contesto italiano. Basti pensare che il progetto di riforma costituzionale del 1962 per introdurre l'elezione diretta del capo dello Stato compattò un fronte d'oppositori che comprendeva quasi tutti i partiti, salvo l'Union pour la Nouvelle République (UNR), secondo movimento gollista dopo l'esperienza del Rpf tra il 1947 e il $1955^{22}$. E tuttavia l'antigollismo italiano traeva alimento anche da motivi slegati dalle idee costituzionali del Generale. Innanzitutto l'ostilità diplomatica di de Gaulle per l'Italia, che era servita a vendicare l'attacco fascista alla Francia del I940 e che condizionò l'ultimo scorcio della guerra e le negoziazioni per il trattato di pace. La cessione di Briga e Tenda a mo' di risarcimento per l'invasione italiana del 1940 non fu il miglior viatico per la ripresa delle relazioni tra i due Paesi. A ciò si aggiunga il fatto che de Gaulle appariva come l'incarnazione di un'idea di Francia legata nostalgicamente alle glorie colonialiste. La stessa concezione gollista dell'integrazione europea faceva capo a un'idea di Stato-Nazione che affondava le radici nell'Ottocento e nella politica di potenza; laddove l'Italia, come noto, puntava a un'organizzazione sovranazionale di tipo federativo sotto l'egida degli Usa e della $\mathrm{Nato}^{23}$. Se si pensa al prevalente orientamento federalista dei prin-

22. R. Brizzi, Delegittimazioni del Generale de Gaulle nella Francia della V Repubblica, in F. Cammarano e S. Cavazza (a cura di), Il nemico in politica. La delegittimazione dell'avversario nell'Europa contemporanea, Bologna, Il Mulino, 20Io, pp. I7I-200.

23. Osservazioni sull'incompatibilità tra la visione europea di de Gaulle e quella dell'Italia sono in M. Vaïsse, De Gaulle, l'Italie et le projet d'Union politique européenne. I958-1963: chronique d'un échec annoncé, "Revue d'histoire moderne et contemporaine», vol. 42, $\mathrm{n}^{\circ}$ 4, 1995, pp. 658-669. 
cipali decisori della politica italiana, si ha la misura di quanto distante questa fosse dalla sensibilità europeista del Generale, tra gli artefici della bocciatura della Ced nel 1954 e fautore di aperture verso l'Urss e di un problematico rapporto con l'atlantismo che mal si conciliavano con i principi della politica estera italiana. Si consideri poi che nel 1958 usciva per i tipi dell'Einaudi la traduzione italiana del libro di Henri Alleg sulla tortura in Algeria $^{24}$, che certo alimentò i timori italiani che de Gaulle proseguisse o accentuasse la politica repressiva dei governi della Quarta Repubblica in Algeria cavalcando le passioni delle sacche retrive del nazionalismo francese. Anche dopo la firma del trattato di Evian nel 1962, le inquietudini circa i sentimenti nazionalisti di de Gaulle stimolavano il terzomondismo dell'Italia, che aveva perduto le proprie colonie dopo la Seconda guerra mondiale e aspirava a divenire mediatrice intercontinentale nel bacino mediterraneo ${ }^{25}$.

Verso la fine degli anni Sessanta, proprio mentre l'astro di de Gaulle declinava, s'intravedeva qualcosa di nuovo negli ambienti della Dc, dove il gruppo di Europa settanta guidato da Bartolo Ciccardini diffondeva una piattaforma programmatica che conteneva tesi apertamente presidenzialiste. Per la prima volta in un partito dell'arco costituzionale si affacciavano idee eretiche rispetto al parlamentarismo di prammatica. Questo costerà a Ciccardini l'aura di simpatizzante dei regimi autoritari e perfino il sospetto di lavorare a trame eversive di tipo golpista. Ammiratori di de Gaulle e in contatto con Pacciardi, gli esponenti di Europa settanta erano in realtà originali interpreti di un'idea di Stato che troverà affermazione di lì a circa vent'anni. In particolare, essi proponevano di temperare il centralismo statalista con un sistema diffuso di elezioni dirette a livello locale che enfatizzava il peso delle autonomie e del decentramento; l'insistenza sulla funzione democratica del referendum avrà sviluppi all'inizio degli anni Novanta, quando uno dei protagonisti di Europa settanta, Mariotto Segni, sarà fautore del rilancio di quest'istituto politico. Similmente fu per l'elezione diretta dei sindaci, che venne introdotta nel 1993 e che era un cavallo di battaglia di Europa settanta.

Nel corso degli anni Settanta la memoria della Resistenza tornava a essere un elemento di primo piano del dibattito pubblico in Italia e uno strumento coesivo nella costruzione dell'identità nazionale. I partiti fedeli

24. Henri Alleg, La tortura, con uno scritto di Jean-Paul Sartre, Torino, Einaudi, 1958. La versione originale è: La Question, Paris, Éd. de Minuit, 1958; per una storia editoriale del libro, si veda A. Berchadsky, "La Question" d'Henri Alleg, un livre-événement dans la France en guerre d'Algérie, Paris, Larousse, 1994.

25. Su quest'ultimo punto cfr. B. Bagnato, L'Italia e la guerra d'Algeria (I954-I962), Soveria Mannelli, Rubbettino, 2012. 
alla Costituzione, tra cui anche il Pci, celebravano l'antifascismo come quintessenza della democrazia contro il terrorismo rosso e nero che minacciava le istituzioni repubblicane. In questo clima di solidarietà nazionale l'immagine degli eroi della Resistenza europea recuperava tutto il suo prestigio e la figura di de Gaulle conobbe un ritorno d'attrattiva negli ambienti più sensibili all'eredità della lotta antifascista e partigiana. È un'evoluzione che s'intreccia strettamente con la biografia di una personalità controversa come Edgardo Sogno. Ex partigiano monarchicoliberale, diplomatico, anticomunista e molto altro, Sogno fondava nel I97I i Comitati di resistenza democratica, centri di propaganda presidenzialista per la creazione di una «seconda repubblica» ${ }^{26}$. Si trattava dell'esito più visibile e presentabile di un disegno volto a impedire l'ascesa al governo del Pci, in cui Sogno individuava il punto di non ritorno per le sorti della fragile democrazia nazionale. Per evitare questo paventato sbocco, Sogno immaginava quel che il magistrato Luciano Violante chiamerà in seguito un "golpe bianco» ${ }^{27}$, ovvero un colpo di Stato possibilmente incruento, tale da aprire la strada a una riforma costituzionale. Il tutto con l'avallo d'influenti ambienti militari che avrebbero dovuto garantire il buon esito dell'operazione.

Nello stesso torno di tempo, novità rilevanti emergevano anche in seno al Psi. Nel 1978 usciva un quaderno di «Mondo Operaio», la rivista ufficiale del partito, consacrato alle ipotesi di «riforma dello Stato» ${ }^{28}$. Il saggio d'apertura scritto da Giuliano Amato, che già dal 1977 era impegnato su questo fronte, si diffondeva su un'analisi delle debolezze della costituzione materiale e sui mezzi per sanarle. Circa l'opportunità di un «esecutivo stabile», Amato riconosceva che «soluzioni di tipo presidenziale» erano poco percorribili in Italia, ma ipotizzava che un eventuale «variante di tale modello", con elezione diretta di capo dello Stato e governo, potesse determinare un sufficiente bilanciamento dei due organi, rafforzandoli e facendoli «derivare entrambi da uno stesso contesto di confronto politico». $\mathrm{Si}$ affacciava in controluce il modello della Quinta Repubblica del 1958 come possibile via d'uscita dallo stallo della 'solidarietà nazionale' e dal compromesso storico tra Dc e Pci, che stava isolando il Psi e accentuando il carattere centripeto dei governi repubblicani: ovvero la tendenza degli

26. E. Sogno, La seconda repubblica, Firenze, Sansoni, I974.

27. La formula fu fatta propria dal diretto interessato: E. Sogno, Il golpe bianco, Milano, Edizioni dello Scorpione, 1978. Cfr. anche l'intervista a Sogno pubblicata postuma: E. Sogno, Testamento di un anticomunista. Dalla Resistenza al golpe bianco, a cura di A. Cazzullo, Milano, Mondadori, 2000.

28. G. Amato, Riforma dello Stato e alternativa della sinistra, in Quale riforma dello Stato?, "Quaderni di Mondoperaio", 1978, p. 24. 
esecutivi a raccogliere intorno alla Dc il contributo dei partiti compatibili con funzioni governative, ferma restando l'egemonia democristiana nella gestione del potere politico.

Fu solo a partire dagli anni Ottanta che la Quinta Repubblica e il suo fondatore godettero di una rivalutazione complessiva favorita da vari fattori. In primo luogo, la fine dei cosiddetti 'anni di piombo' in Italia e quindi l'avvento di una stagione propizia a ipotesi di riforma delle istituzioni, dopo una lunga fase in cui la difesa delle stesse istituzioni era stata prioritaria; in secondo luogo, la vittoria di François Mitterrand alle elezioni del 198I realizzava l'alternanza ai vertici delle istituzioni francesi, dimostrando la funzionalità e l'efficacia pluralista dell'assetto istituzionale creato oltralpe tra fine anni Cinquanta e inizio anni Sessanta; infine l'ascesa al potere di Bettino Craxi, leader del Psi dal 1976, capo del governo dal 1983 al 1987, fautore di una concezione personalista e carismatica del potere. Questa svolta si accompagnava a un approccio meno pregiudiziale allo studio della Quinta Repubblica di cui sono prova gli atti del convegno dell'École française de Rome tenutosi nel 1980 e centrato su una comparazione tra le Costituzioni italiana del 1948 e francese del $1962^{29}$.

Nonostante questa mutata temperie e il sostegno intellettuale di personalità eminenti del mondo giuridico e politologico — si pensi rispettivamente a Enzo Cheli e Luciano Cavalli - l'idea d'innestare nel parlamentarismo italiano i germi del presidenzialismo francese non troverà un inquadramento progettuale preciso. Il Psi continuò ad essere il partito più vivace sul fronte delle ipotesi di riforma delle istituzioni in senso presidenziale, ma rimanevano, quelle socialiste, istanze minoritarie che non incisero significativamente sui lavori delle due commissioni per la riforma dello Stato: la prima Bicamerale (1983-1985) e la seconda (I992-1994). La fine della Guerra fredda, l'inchiesta Mani Pulite, il crollo del sistema dei partiti della Prima Repubblica creavano le basi perché si facesse largo un nuovo modo di guardare alle istituzioni italiane. Il parlamentarismo entrava definitivamente in crisi e il rafforzamento del potere decisionale diventava una priorità nell'agenda delle riforme. Il presidenzialismo non era più un tabù e questo vento di cambiamento si ripercuoteva anche sulla letteratura scientifica ${ }^{30}$.

29. Vedi per esempio G. Andreotti et al., Deux constitutions: la Ve République et la République italienne. Parallèles et commentaires, actes du colloque de Rome ( $\mathrm{I}^{\mathrm{er}}-3$ décembre 1980 ), Rome, École française de Rome, 1988.

30. Cfr. ad esempio S. Gentile, Capo carismatico e democrazia. Il caso de Gaulle, Milano, FrancoAngeli, I998; G. Quagliariello, De Gaulle e il gollismo, Il Mulino, Bologna, 2003; R. Brizzi e M. Marchi, Charles de Gaulle, Bologna, Il Mulino, 2008. Osservazioni intorno alla monografia di Quagliariello nel contesto della storiografia 
In conclusione, la breve disamina condotta fin qui mostra come de Gaulle abbia costituito un costante elemento di confronto per le élites politiche italiane e una fonte di studio per gli addetti ai lavori delle scienze storiche e politologiche. La ragione di quest'interesse risiede certo nell'eccezionalità intrinseca dell'uomo ma anche nell'attenzione che tradizionalmente gli osservatori italiani hanno avuto per la scena francese, considerata una sorta di paradigma della teoria e della prassi democratiche e dunque una fonte d'ispirazione e talora un modello di riferimento. In questo senso, il ritorno di de Gaulle al potere nel 1958 creò una soluzione di continuità percepita da Roma come la fine di un periodo, quello del dopoguerra, in cui l'Italia si era avvicinata come mai prima di allora all'esempio politico-costituzionale francese. De Gaulle, di fatto, allontanava la Francia dal modello parlamentare introiettato dagli italiani ma accendeva anche curiosità per gli esiti di un esperimento inedito e per certi versi temerario. Per decenni, tuttavia, anche i più convinti sostenitori del presidenzialismo e oppositori della 'partitocrazia' guardarono più al modello statunitense che a quello transalpino. Quando finalmente i tempi furono maturi perché in Italia si potesse pensare al gollismo con meno pregiudizi ideologici, il caso francese tornò a essere un esempio fertile, potenzialmente risolutivo per i problemi nostrani, fino a diventare un modello forse esportabile alle nostre latitudini. Al di là delle contingenze politiche, emergono nell'ambito in questione due tendenze di lungo periodo: l'attrazione degli italiani per le cose di Francia, soprattutto in campo politico e istituzionale; l'influenza di de Gaulle nell'immaginario collettivo e la sua sopravvivenza simbolica. È lecito pensare che questi due elementi continueranno ad alimentare lo studio e l'uso' pubblico del gollismo come fenomeno imprescindibile della contemporaneità, quasi l'idealtipo di una concezione del potere forse squilibrata sul piano istituzionale ma altrettanto suggestiva per carisma e potenza iconica.

francese sul gollismo sono in L. Bonfreschi e C. Vodovar, La transizione francese del I958 nella storiografia degli ultimi anni, «Ventunesimo secolo», $\mathrm{n}^{\circ}$ 23, ottobre 20IO, p. IO7-I23, soprattutto pp. III-II8. 